\title{
Weak violation of universality for Polyelectrolyte Chains: Variational Theory and Simulations
}

\author{
Gabriele Migliorini ${ }^{1}$, Vakhtang G. Rostiashvili ${ }^{1}$, and Thomas A. Vilgis ${ }^{1,2}$ \\ ${ }^{1}$ Max Planck Institute for Polymer Research \\ 10 Ackermannweg, 55128 Mainz, Germany. \\ 2 Laboratoire Européen Associé, Institute Charles Sadron \\ 6 rue Boussingault, 67083 Strasbourg Cedex, France.
}

(November 20, 2018)

\begin{abstract}
A variational approach is considered to calculate the free energy and the conformational properties of a polyelectrolyte chain in $d$ dimensions. We consider in detail the case of pure Coulombic interactions between the monomers, when screening is not present, in order to compute the end-to-end distance and the asymptotic properties of the chain as a function of the polymer chain length $N$. We find $R \simeq N^{\nu}(\log N)^{\gamma}$ where $\nu=\frac{3}{\lambda+2}$ and $\lambda$ is the exponent which characterize the long-range interaction $U \propto 1 / r^{\lambda}$. The exponent $\gamma$ is shown to be non-universal, depending on the strength of the Coulomb interaction. We check our findings, by a direct numerical minimization of the variational energy for chains of increasing size $2^{4}<N<2^{15}$. The electrostatic blob picture, expected for small enough values of the interaction strength, is quantitatively described by the variational approach. We perform a Monte Carlo simulation for chains of length $2^{4}<N<2^{10}$. The non universal behavior of the exponent $\gamma$ previously derived within the variational method, is also confirmed by the simulation results. Non-universal behavior is found for a polyelectrolyte chain in $d=3$ dimension. Particular attention is devoted to the homopolymer chain problem, when short range contact interactions are present.
\end{abstract}

\section{INTRODUCTION}

Charged polymers play a central role in theory and experiments. Polyelectrolytes are macromolecules containing ionizable groups. They dissociate in water to form charged groups and low-molecularweight counter ions. It is important to remark that interactions between different polyions in a many chain system are of crucial importance for any realistic system with a low but finite overlap concentration. The problem of the single chain conformation is the one of the essential questions for polyelectrolyte solutions and has been under investigation in the recent years. This will be the topic of the present paper as well, but in a more general context. As common to most of the recent investigation, we consider the limit of infinite dilution and zero salt concentration for polyelectrolytes. In this limit a polyelectrolyte can be represented as a connected sequence of charged and uncharged monomers in a "dielectric vacuum" that constitutes the solvent. ¿From a scaling point of view, the properties of single polyelectrolyte chains, despite the long time debates are still not completely clear. Indeed, for the neutral polymer chain system, where a clear understanding of the scaling properties has been achieved [1], the details of the local chain structure are not essential, while in the polyelectrolyte chain problem they could be important [2,3]. In particular the long range nature of the Coulomb interaction couples via the presence of the solvent, the short and long length scales of the system . Thus a scaling approach to polyelectrolytes results to be more involved than for neutral chains. The interaction between monomers, counterions and solvent molecules leads to the important phenomenon of counter ion condensation [4] and a realistic description should take into account all the different characters of this scenario. On the other hand, a minimal or bare essential description of the system is required in order to achieve a quantitative description of the single polyelectrolyte chain problem.

In this paper we will present a generalized form of des Cloizeaux Gaussian variational method [5,6] to study the scaling behavior and local properties of polyelectrolytes. Variational techniques have been shown to be well suited to describe a self-interacting polymer with long range repulsion [7]. Scaling properties of the end-to-end distance of the chain have been discussed for the long - ranged intra-molecular interaction $1 / r^{\lambda}$ at an arbitrary spatial dimensionality $d$. A recent numerical implementation of such a variational technique, for the case of Coulombic interaction (when $\lambda=d-2$ ), seems to be promising as well [8]. On the other hand, the validity of the variational approach for short range interacting homopolymer chains must be questioned [7 90 for natural and well known reasons. The most studied example in the context of polymer physics is the problem of the self avoiding chain. It was proven that the problem shows universality in the limit of infinitely long chains 1] and can be treated by field theoretic 
methods common to phase transitions. Indeed the renormalization group (RG) theory manifests these statements and develops systematic methods to compute the scaling exponents, e.g. for the size of the chain [6]. The variational technique provides an alternative approach since most of the approximations can be controlled. Although, it is well known from elementary statistical mechanics that the variational technique cannot provide such powerful tool due to their mean - field character. Therefore this technique is expected to work in the mean field limit, i.e. at large dimensions (at the upper critical dimension) or for long range potentials. Thus it is well investigated and generally believed that the Gaussian variational approach is better suited for long-range interacting chains.

Moreover, it has been suggested that in the study of random heteropolymer 10,11 and directed polymer in a random media [12], a variational approach in replica space can be also successfully applied. We postpone these issues for future investigations. Here we will mainly concentrate on the pure long-range and short-range homopolymer problems within the Gaussian variational technique.

In section II we introduce the Gaussian polymer model and calculate the variational free energy using a general Gaussian trial Hamiltonian in the spirit of des Cloizeaux 15,6] and reference [13]. We then critically review the asymptotic analysis given originally in reference 阿 and suggest a new one which leads to the Flory exponents corrected by the presence of logarithmic factors. In section III we describe a numerical technique in order to solve the Euler equations corresponding to the variational principle, by means of a new algorithm specially devised for this problem. An independent algorithm, that simply minimizes the variational free energy is shown to be quantitatively equivalent to the direct solution of the Euler equations. The numerical solution of the Euler equations is then carefully analyzed for polymer chains of increasing size. We show here that our new asymptotic analysis is in quantitative agreement with our numerical findings. We discuss connections with the short range homopolymer problem within the Gaussian Variational Principle and results for the end-to-end distance are obtained. In section IV Monte Carlo simulation results, obtained by means of a pivot algorithm, are presented, showing quantitative agreement with our variational analysis. The case of a self-interacting homopolymer chain, when an attractive two-body interaction between the monomers is considered together with a three body repulsive term, can also be studied within our variational approach. Preliminary calculations shows that the globular formation, expected from simple phenomenological arguments and due to the balancing between the two body and three body interactions, takes place. The instability of the globule state due to a Coulombic repulsion between the monomers is also described by our variational principle and will be presented elsewhere [14]. Conclusions and further prospects are summarized in section V.

\section{THE GAUSSIAN VARIATIONAL PRINCIPLE}

\section{A. Basic equations}

First we start from a well known example for a Gaussian variational approach suitable for the investigations presented below. We introduce a simple problem to compute the free energy of a self-interacting polymer chain with Coulombic repulsion between the monomers, based on a discrete representation of a chain of $N$ monomers. The method relies on the well known variational principle, where a Gaussian trial probability is proportional to the exponential of a quadratic form of the monomer coordinates $\overrightarrow{r_{j}}$,

$$
P_{V}\left(\overrightarrow{r_{1}}, . ., \overrightarrow{r_{N}}\right)=Z_{V}^{-1} \exp \left\{-H_{0}\left(\overrightarrow{r_{1}}, . ., \overrightarrow{r_{N}}\right)\right\} .
$$

In eq.(2.1) the trial Hamiltonian $H_{0}$ is given by

$$
H_{0}\left(\overrightarrow{r_{1}}, . ., \overrightarrow{r_{N}}\right)=\frac{d}{2} \sum_{i=1}^{N} \sum_{j=1}^{N} G_{N}(i-j)\left(\overrightarrow{r_{i}}-\overrightarrow{r_{j}}\right)^{2}
$$

and $Z_{V}$ is a normalization constant determined by the condition

$$
\int P_{V}\left(\overrightarrow{r_{1}}, . ., \overrightarrow{r_{N}}\right) d^{d} \overrightarrow{r_{1}} \ldots d^{d} \overrightarrow{r_{N}}=1 .
$$

Up to this point different forms of the Gaussian variational principle could be considered. We decided here to consider the variational form originally proposed by des Cloizeaux [5] in the context of the selfavoiding homopolymer problem. The main essential features of the Gaussian variational principle can be obtained within this choice of the variational form according to equation (2.2). More sophisticated 
forms of the variational kernel including a classical path $\overrightarrow{r_{0}}$ and eventual anisotropy along parallel and perpendicular directions to the polymer backbone, can be also included [13]. By the appropriate choice of a periodic boundary conditions for the chain the quadratic form $H_{0}$ can be diagonalized by introducing cyclic coordinates (Rouse modes) for the monomer positions

$$
\begin{aligned}
& \overrightarrow{\rho_{q}}=N^{-\frac{1}{2}} \sum_{j=1}^{N} \exp [\mathrm{i} 2 \pi j q / N] \overrightarrow{r_{j}} \\
& \overrightarrow{r_{j}}=N^{-\frac{1}{2}} \sum_{q=1}^{N} \exp [-\mathrm{i} 2 \pi j q / N] \overrightarrow{\rho_{q}} .
\end{aligned}
$$

Indeed, due to cyclic invariance the Cartesian components of $\vec{\rho}_{q}$ satisfy

$$
\left\langle\rho_{q}^{(j)} \rho_{q^{\prime}}^{\left(j^{\prime}\right)}\right\rangle=\frac{1}{d} \delta_{j j^{\prime}} \delta_{q q^{\prime}} g_{N}^{-1}(2 \pi q / N),
$$

where $g_{N}^{-1}(2 \pi q / N)$ is positive and discontinuous function of $k=2 \pi q / N$ and is related to the Gaussian propagator $G$ of equation (2.2) via the following expression

$$
g_{N}(2 \pi q / N)=\sum_{n=1}^{N-1} G_{N}(n)[1-\cos (2 \pi q n / N)]
$$

The variational free energy for a uniformly charged polymer of polymerization index $N$ in a $d$ dimensional space can be computed according to the usual Feynman inequality

$$
F \leq F_{V}=\left\langle H-H_{0}\right\rangle_{0}+F_{0},
$$

where $F_{0}$ is the free energy of the Gaussian model defined by equation (2.2). In equation (2.7) $H$ represents the full Hamiltonian of a charged polymer chain with a pure long - range form for the monomer-tomonomer interaction,

$$
V\left(\left|\overrightarrow{r_{i}}-\overrightarrow{r_{j}}\right|\right)=\left|\overrightarrow{r_{i}}-\overrightarrow{r_{j}}\right|^{-\lambda},
$$

where $\lambda<d$. The expression for the Hamiltonian reads

$$
H=\frac{d}{2 b^{2}} \sum_{i=1}^{N}\left(\overrightarrow{r_{i+1}}-\overrightarrow{r_{i}}\right)^{2}+\beta / 2 \sum_{i=1}^{N} \sum_{j=1}^{N} V\left(\left|\overrightarrow{r_{i}}-\overrightarrow{r_{j}}\right|\right),
$$

where $\beta=l_{\mathrm{B}}^{\lambda} / A^{2}$ depends on the Bjerrum length $l_{\mathrm{B}}=\left(e^{2} / 4 \pi \epsilon k_{B} T\right)$ and where the distance between charged monomers along the chain is given by $A$ in units of the Kuhn segment $b$. $A$ defines thus the charge fraction $f=1 / A$.

It is straightforward to derive the following expression for the variational free energy $F_{V}$ in the limit of infinitely long chain $N \rightarrow \infty$,

$$
\begin{aligned}
& F_{V}[g(k)] / N=\frac{d}{2} b(1)+\beta\left(\frac{d}{2}\right)^{\frac{\lambda}{2}} \frac{\Gamma\left(\frac{d-\lambda}{2}\right)}{\Gamma\left(\frac{d}{2}\right)} \sum_{n=1}^{\infty}[b(n)]^{-\frac{\lambda}{2}}+ \\
& \frac{d}{4 \pi} \int_{-\pi}^{+\pi} \log g(k) d k-\frac{d}{2}[1+\log (2 \pi / d)],
\end{aligned}
$$

where

$$
\begin{gathered}
b_{N}(i-j)=\frac{1}{N} \int_{-\pi}^{+\pi} \frac{(1-\cos ((i-j) k))}{g(k)} d k, \\
b_{N}(i-j) \equiv\left\langle\left(\overrightarrow{r_{i}}-\overrightarrow{r_{j}}\right)^{2}\right\rangle
\end{gathered}
$$

and 


$$
\lim _{N \rightarrow \infty} g_{N}\left(k_{N}\right) \equiv g(k), \quad g(k) \geq 0 .
$$

The function $g(k)$ satisfies the symmetry requirements

$$
g(k)=g(-k), \quad g(k+2 \pi)=g(k) .
$$

Minimization of the variational free energy $F_{V}$ yields

$$
\begin{aligned}
g(k)=1 & -\cos (k)-\beta\left(\frac{\lambda}{2}\right)\left(\frac{d}{2}\right)^{\frac{\lambda}{2}} \frac{\Gamma\left(\frac{d-\lambda}{2}\right)}{\Gamma\left(\frac{d}{2}\right)} \\
& \times \sum_{n=1}^{\infty}(1-\cos (n k))[b(n)]^{-\frac{\lambda+2}{2}} .
\end{aligned}
$$

Equations (2.10)-(2.15) represent the generalization of the Gaussian variational principle for the longrange potential (2.8) which was introduced first in reference 77. For the pure Coulombic case $\lambda=d-2$, comparing the expression for the variational free energy (2.10) with the correspondent expression for the short range interaction problem [5],

$$
\begin{gathered}
F_{V}[g(k)] / N=\frac{d}{2} b(1)+w \sum_{n=1}^{\infty}[b(n)]^{-\frac{d}{2}}+ \\
\frac{d}{4 \pi} \int_{-\pi}^{+\pi} \log g(k) d k-\frac{d}{2}[1+\log (2 \pi / d)],
\end{gathered}
$$

leads to the following conclusion which holds within the Gaussian Variational Principle. All universal exponents for the Coulombic case are related to the corresponding exponents for the short-range interaction case through the dimensional shift

$$
d \rightarrow d-2 .
$$

The result of minimization of equation (2.16) reads

$$
g(k)=1-\cos (k)-w \sum_{n=1}^{\infty}(1-\cos (n k))[b(n)]^{-\frac{d+2}{2}},
$$

which is of the same form like equation (2.15).

Before moving to the next section, where a careful numerical implementation of the Euler equations (2.11)-(2.15) for polymer chains of increasing length $N$ is presented, we revise the asymptotic analysis of the Euler equations in the limit of small $k \rightarrow 0$, which was originally given by des Cloizeaux [5]. The behavior of $g(k)$ at small $k$ determines the asymptotic behavior of $b(n)$ for $n>>1$ via equation (2.11). Let us assume first that in this limit $g(k)$ has the following form

$$
g(k) \simeq g|k|^{1+2 \nu}, \frac{1}{2}<\nu<1,
$$

then the correlation function $b(n)$, reads

$$
b(n) \simeq b n^{2 \nu} .
$$

We will now proceed to analyze the Euler equations in the scaling limit of small $k$ values within this simple ansatz.

\section{B. The asymptotic analysis - neutral chains}

To demonstrate several problems within the Gaussian variational principle we are going to rewise in this section the asymptotic analysis of the Euler equation (2.18) for neutral chains, which was originally given in the context of the self avoiding chain problem [5], and later extended to self interacting polymer chains with long-ranged Coulomb like interactions [7]. It has been suggested that due to the long ranged nature of the interactions between the monomers the Gaussian Variational Principle is particularly well 
suited to describe the asymptotic behavior of polyelectrolyte chains. Special attention has also been devoted [8,13,15] to the case of screened Coulombic interaction, within the Debye Hückel approximation.

Let us consider the interaction term

$$
I(k)=w \sum_{n=1}^{\infty}(1-\cos (n k))[b(n)]^{-\frac{d+2}{2}} .
$$

We can obtain the asymptotic expression for $I(k)$ in the $k \rightarrow 0$ limit, substituting the series with an integral [5] and assuming that the dominant contribution would correspond to large values of $n$. The result can be written in the form

$$
I(k) \simeq-w \frac{\pi b^{-\frac{d+2}{2}}}{2 \Gamma(\delta) \cos \left(\frac{\pi}{2} \delta\right)} k^{\delta-1}+w b^{-\frac{d+2}{2}} \zeta(\delta-2) \frac{k^{2}}{2}
$$

where $\delta=\nu(d+2)$.

An alternative derivation can be obtained in the asymptotic regime $n>>1$ evaluating exactly the series

$$
S(\delta, z)=\sum_{n=1}^{\infty} \frac{z^{n}}{n^{\delta}}
$$

at $z=\exp (i k)$. The explicite calculations are given in Appendix where it is stressed that we should be distinguish between noninteger and integer $\delta$. We apply these results for calculation of eq. (2.21) where $b(n)$ is given by eq.(2.20).In this case we find

$$
I(k) \simeq w b^{-\frac{d+2}{2}}[S(\delta, z=1)-\operatorname{Re} S(\delta, z)],
$$

where $\delta=\nu(d+2)$. Considering non-integer values of the exponent $\delta$ (this assumption will be checked afterwards) eq. (5.2) yields

$$
I(k)=w b^{-\frac{d+2}{2}}\left[-\frac{\pi}{2 \Gamma(\delta) \cos \left(\frac{\pi}{2} \delta\right)} k^{\delta-1}-\sum_{p=1}^{\infty} \zeta(\delta-2 p)(-1)^{p} \frac{(k)^{2 p}}{(2 p) !}\right] .
$$

This equation generalizes eq. (2.22) ( see also eq. (B.9) of ref. [5] ). The Euler equation (2.18) together with the expansion (2.25) gives, in the $k \rightarrow 0$ limit

$$
g k^{1+2 \nu}=\frac{1}{2} k^{2}-w b^{-\frac{d+2}{2}} \zeta(\delta-2) \frac{k^{2}}{2}+w \frac{\pi b^{-\frac{d+2}{2}}}{2 \Gamma(\delta) \cos \left(\frac{\pi}{2} \delta\right)} k^{\delta-1}+O\left(k^{4}\right),
$$

where $\delta=\nu(d+2)$.

Let us impose first the balancing conditions in the spirit of des Cloizeaux: (i) the first two terms on the r.h.s. balance each other, (ii) the term on the l.h.s. and the third term on the r.h.s. are also balanced. Then we immediately obtain

$$
\nu=\frac{2}{d}
$$

as a valid exponent, but together with the additional conditions

$$
\begin{aligned}
& w b^{-\frac{d+2}{2}} \zeta(\delta-2)=1 \\
& g=\frac{w \pi b^{-\frac{d+2}{2}}}{2 \Gamma(\delta) \cos \left(\frac{\pi}{2} \delta\right)}
\end{aligned}
$$

contained in the above equations, the physical interpretation becomes, however, more complicated. Moreover, we must add to these equations the general relation between $g$ and $b$, which originates from equation (2.11)

$$
b=\frac{1}{2 g \Gamma(1+2 \nu) \sin (\pi \nu)} .
$$


Equation (2.27) is thus the well known result for the exponent $\nu$, whereas equations (2.28)-(2.30) set up three equations for the two unknowns $b, g$. Equations (2.28)-(2.30) determines not only the amplitudes $g$ and $b$ but also fixes the value of the interaction parameter $w$ itself. This delicate point, (see equation (IV.52) of reference [5]) has already been discussed by Allegra [9]. This is contradictory in the sense that generally the interaction parameter $w$ is an arbitrary quantity. The proper balance condition must consist of a single equation, whereas the conditions (2.28)-(2.29), derived in the asymptotic analysis of des Cloizeaux, overconstrains the parameters involved. Besides that the parameter $\delta=2(d+2) / d$ is only non-integer for $d=3$ and is integer for $d=1,2,4$ (where the Flory scaling produces exact results). This indicates that the des Cloizeaux solution can not be valid in this later case since it relies on the expansion (2.25) which (see Appendix) is only valid for non-integer values of $\delta$.

In order to avoid the overconstraints we try to impose the Flory balancing conditions : (i) the elastic term, which is proportional to $k^{2}$, is balanced by the interaction term eq.2.21), (ii) the term on the l.h.s. (or the entropic term) is negligible compare to the elastic and interaction terms on the r.h.s. Obviously the Flory balancing conditions have one constraint less. In this case for $S(\delta, z)$ we must use eq.(5.5) where $\delta=m=\nu(d+2)=3$ and $z=\exp (i k)$. This immediately leads to the well known Flory exponent $\nu_{\mathrm{F}}=3 /(d+2)$. Then the interaction term 2.21) becomes

$$
\begin{aligned}
& I(k)=\lim _{k \rightarrow 0} w b^{-\frac{d+2}{2}}[S(m=3, z=1)-\operatorname{Re} S(m=3, z)]= \\
& w b^{-\frac{d+2}{2}} \sum_{n=1}^{\infty} \frac{1-\cos (n k)}{n^{3}} \simeq w b^{-\frac{d+2}{2}}[\psi(3)-\psi(1)-\log k] \frac{k^{2}}{2}+\mathcal{O}\left(k^{4}\right) .
\end{aligned}
$$

The resulting Euler equation takes the form

$$
g k^{1+2 \nu_{\mathrm{F}}}=\frac{1}{2} k^{2}-w b^{-\frac{d+2}{2}}[\psi(3)-\psi(1)-\log k] \frac{k^{2}}{2}+O\left(k^{4}\right) .
$$

Eq.(2.32) can be also obtained directly from eq.(2.26) if we assume $\delta=3+\epsilon$, where $\epsilon \rightarrow 0$. Then the poles which have its origin from the second and third terms on the r. h. s. are canceled (see e.g. Appendix and sec. 1.11 in ref. [16]) but the term $k^{2} \log k$ appears. The presence of the $k^{2} \log k$ term in equation (2.32) leads to the conclusion that the pure power law ansatz $g(k) \propto k^{1+2 \nu}$ (or $b(n) \propto n^{2 \nu}$ ) does not solve in the asymptotic limit $k \rightarrow 0$ the Euler equation (2.18) once the exponent $\delta=(d+2) \nu$ is assumed to be integer. In fact, the direct numerical solution of the Euler equations (2.15) and (2.18) presented in the next section does not show a pure power law behavior in the asymptotic regime $k<<1$. We also see that the term $k^{2} \log k$ naturally appears in the previous expressions for the series $S(\delta, k)$, which suggests to consider logarithmic corrections in the initial ansatz for $b(n)$. This will allow to stay within a Flory-like solution (corresponding to the integer value $\delta=\nu(d+2)=3$ ) and also to compute explicitly the exponent that characterize the logarithmic correction to the pure power law scaling behavior. Moreover the problem of having three equations for the two parameters $g$ and $b$, which invalidates the asymptotic analysis of des Cloizeaux, is naturally solved within the new ansatz we propose in the next subsection. The value of $w$ is no longer constrained to a certain value as it should be the case in an ideal experiment where it is allowed to tune the Coulombic interaction (or the quality of the solvent for the self avoiding problem).

\section{Corrections to scaling}

As it was suggested we will stay within the Flory balancing condition but try the next asymptotic ansatz for the correlation function (2.12)

$$
b(n) \simeq b_{0} n^{2 \nu}(\log n)^{2 \gamma},
$$

where $\gamma$ is the exponent that characterize the logarithmic correction to scaling. Substituting the ansatz (2.33) in the Euler equation (2.18) gives for the interaction term

$$
I(k)=w b_{0}^{-\frac{d+2}{2}} \sum_{n=1}^{\infty} \frac{1-\cos (n k)}{n^{\nu(d+2)}[\log n]^{\gamma(d+2)}} .
$$

The largest contribution to this series at small $k$ comes from the large $n$ limit. We can substitute the sum with an integral (for the power law ansatz, this is explained and justified in the Appendix) to obtain 


$$
I(k) \simeq w b_{0}^{-\frac{d+2}{2}} \int_{\bar{n}}^{\infty} d n \frac{1-\cos (n k)}{n^{\nu(d+2)}[\log n]^{\gamma(d+2)}},
$$

where $\bar{n}$ is a cutoff we introduce to regularize the integral. Equation (2.35), after substituting $n=x / k$ reads

$$
I(k) \simeq k^{\nu(d+2)-1} w b_{0}^{-\frac{d+2}{2}} \int_{\bar{x}}^{\infty} d x \frac{1-\cos (x)}{x^{\nu(d+2)}[\log x-\log k]^{\gamma(d+2)}},
$$

where, because of the Flory balancing conditions $\left(I(k) \propto k^{2}\right)$, we should impose $\nu(d+2)=3$. Let us consider a value $t$ such that at $x<t$ it is ensured that $1-\cos x \simeq x^{2} / 2$ holds. In this case the integral in equation (2.36) becomes

$$
\begin{aligned}
J(k) & =\int_{\bar{x}}^{\infty} d x \frac{1-\cos (x)}{x^{3}[\log x-\log k]^{\gamma(d+2)}} \\
& \simeq \frac{1}{2} \int_{k \bar{n}}^{t} d x \frac{1}{x[\log x-\log k]^{\gamma(d+2)}} \\
& +\int_{t}^{\infty} d x \frac{1-\cos (x)}{x^{3}[\log x-\log k]^{\gamma(d+2)}}=J_{1}+J_{2} .
\end{aligned}
$$

At $k \rightarrow 0 J_{2}(k) \rightarrow 0$, while the integral $J_{1}(k)$ defined above can be evaluated as

$$
J_{1}(k)=\frac{1}{2[\gamma(d+2)-1]}\left[[\log (\bar{n})]^{1-\gamma(d+2)}\right],
$$

provided that $\gamma(d+2)>1$ and $\bar{n}>1$. We conclude that the Euler equation (2.18) is properly regularized by the logarithmic ansatz (2.33) and the cutoff parameter $\bar{n}>1$. The balance in equation (2.18), between the elastic energy term and the interaction term, gives

$$
\begin{aligned}
& \nu=\frac{3}{d+2}, \\
& \gamma>\frac{1}{d+2} .
\end{aligned}
$$

\section{Correction to scaling - charged chains}

For the Coulombic case, considering the dimensional shift rule (2.17), we obtain

$$
\begin{aligned}
\nu & =\frac{3}{d}, \\
\gamma & >\frac{1}{d} .
\end{aligned}
$$

The inequality we just derived will be verified numerically in the next section. Values for the exponent $\gamma$ will be obtained via the numerical solution of the Euler equations (2.10)-(2.15) and via Monte Carlo simulations. Within our asymptotic analysis, we can obtain an expression for the exponent $\gamma$ that depends on the interaction strength. In particular by balancing the prefactors of the proper terms we have

$$
\gamma \simeq \frac{1}{d}\left(1+\frac{w}{b_{0}(w)^{d / 2}}\right)
$$

where we also took into account that the amplitude $b_{0}$ can depend from $w$. The exponent $\gamma$ depends on the interaction strength, being non universal. This will be tested against simulations and numerical minimization of the variational free energy (2.10).

It was suggested [3] that above a certain minimum charge fraction $f \simeq N^{-3 / 4}\left(b / l_{\mathrm{B}}\right)^{1 / 2}$ the electrostatic interactions are relevant and the end-to-end distance can be written in the form

$$
R \simeq N f^{2 / 3}\left(l_{\mathrm{B}} b^{2}\right)(\log N)^{\gamma} .
$$


In this equation the value of $\gamma=1 / 3$ is confirmed also by a simplified chain under tension variational method [2, 18] where the trial Hamiltonian is the Hamiltonian of a Gaussian chain subject to an external tension $\vec{F}$. Indeed we also reproduce this result for large enough values of the Coulomb strength as we will discuss in the next section. We observe that the exponent $\gamma$ is non universal, i.e. decreasing with the interaction strength, and approaching the limiting value $\gamma=1 / 3$ from above in the region of $\beta>1$, in agreement with equation (2.42). At $\beta=1$, the value of $\gamma \simeq 0.38$ is in good agreement with the calculation of Söderberg and collaborators [19]. It is important to remark that if we assume in the previous asymptotic analysis that the $\gamma$ exponent is universal a spurious nested logarithmic form for the end-to-end distance (the way Allegra [21] discussed long time ago) is obtained. This scenario however is not confirmed by the numerical analysis of the Euler equations as will be presented in section III. The balancing between the elastic energy and the interaction term determines the exponents (2.39)-(2.40) for the self avoiding chain (or short-range interaction) problem, whereas for the Coulombic case the result is given in equations(2.41)- (2.42).

The term that appears on the l.h.s. of equation $(2.26)$ is negligible in this case. The proper condition for this to happen reads $1+2 \nu>2$, which leads to the condition $d<4$ for the self-avoiding chain [1] and $d<6$ for the Coulombic chain [23]. Accordingly the upper critical dimensionality is $d_{c}=4$ for the former case and $d_{c}=6$ for the latter one. At $d>d_{c}$ we can neglect the interaction term and the chain becomes Gaussian.

We would like to make a general remark at this point. It is clear that the variational technique cannot cover the results of the RG. From the universality of the self avoiding walk problem it is well known that the choice of the parameter $w$ does not change the scaling exponent and below the upper critical dimension no logarithmic corrections are necessary. At the critical dimension, these become obviously real. On the other hand the variational technique produce these corrections naturally and works well in the mean field limit. For the case of polyelectrolytes the situation is more complex. The Coulomb potential changes its nature from long range to short range between three and four dimensions. Therefore the fixed point and scaling behavior is completely different, as it has been discussed already by de Gennes [1]. It is therefore also necessary to check our results by alternative methods.

\section{NUMERICAL SOLUTION OF THE EULER EQUATIONS}

In this section we consider in detail the Euler equations (2.11) - (2.15) for a polymer chain of length $N$ with cyclic boundary conditions, obtained by minimization of the variational free energy $F_{V}$. Two different ways in order to solve the variational principle minimization for a finite chain of length $N$ are possible. On the one side, a multidimensional minimization of the free energy $F_{V}$ in a $N$ dimensional space is considered [17]. On the other side, a direct solution of the Euler equations will be presented.

We checked first that our multidimensional algorithm is able to reproduce the simple Gaussian profile for the simple case of the non interacting chain problem. We then implement our algorithm by choosing a very small value of the interaction strength $\beta$, where the solution is expected to be very close to the simple Gaussian chain solution, and give $N+1$ trial profiles for the function $g_{N}(k)$. The multidimensional algorithm via a sequence of reflections and translations between the $N+1$ trial profiles $g_{N}(k)$, relaxes to the true solution that can be resolved within the numerical precision desired. Once the algorithm was properly tuned, we moved on studying systematically the shape of the optimal profile that minimizes the free energy $F_{V}$ for increasing values of the interaction strength $\beta$. For any specific value of $\beta$ the optimal profile was checked to satisfy the basic symmetry requirements given in equation (2.14). Once the numerical solution $g_{N}(k)$ that minimize the variational free energy was found, we proceeded to analyze the properties of the solution in the asymptotic regime, in order to check our results for the maximal mean square distance $b(N / 2)$. Results for the mean square distance $\left\langle\left(\vec{r}_{i+n}-\vec{r}_{i}\right)^{2}\right\rangle$ for intermediate values of $n$ were also studied in order to confirm the phenomenological predictions of the electrostatic blob picture.

A direct implementation of the equations (2.11) - (2.15) does confirm the multidimensional minimization of the free energy $F_{V}$ and also represents a convenient method to solve the variational problem itself. We also consider, for the case of $\lambda=1$ a Fast Fourier Algorithm that let us consider chains of length up to $N=2^{15}$. The difference between the values of the exponent $\gamma$ computed with $N<1024$ and $N<32768$ are very small and tends to vanish when the interaction strength increases. We consider this as a second, independent test to confirm the non-universal behavior of $\gamma$. For this reason we will consider shorter chains only for what concerns the case of $\lambda=2$ and for the Self Avoiding Chain Problem (see section IIc). We start with a purely Gaussian profile in order to compute the left hand side of equation (2.15), via the Fourier transform of (2.13) that defines the mean square distance $b(n)=\left\langle\left(r_{i+n}-\overrightarrow{r_{i}}\right)^{2}\right\rangle$ for a small value of the interaction strength $\beta$ and iterate this procedure until convergence is achieved. 
Once the solution of the Euler equations is found for a specific value of the interaction, one can use it as a starting point for the following iteration procedure, in order to solve the same set of equations for a higher value of $\beta$. It is important to note that a simple recursive implementation of the Euler equations above is highly unstable in the sense that the number of iterations required as a function of $\beta$ rapidly increases, making a simple iteration procedure quite ineffective. A simple solution to this problem can be found borrowing ideas from the renormalization group literature [24]. Renormalization Group Theory was successfully applied to determine the phase diagram and magnetic properties of disordered spin systems [25]. A functional set of recursion relations for the probability distribution function of bond randomness was studied and a fixed distribution was found for different values of the initial conditions. Instead of a simple iterative procedure, which could be a rather slow converging process, a multidimensional bisection between different initial conditions for the renormalization group trajectory turns out to be a very effective method in order to achieve convergence. In a similar fashion, instead of a simple iteration of the Euler equations above, we prefer to bisect between possible initial profiles of the function $g(k)$ given above.

\section{A. Results for the Coulombic chain}

We obtained the solution of the Euler equations (2.11)- 2.15) via the iterative method discussed above and independently via the direct minimization of the variational free energy $F_{V}$ in equation $(2.10$ ) within the numerical precision. We considered cyclic chains of length $2^{4}<N<2^{15}=32768$, for different values of the interaction strength $\beta$ and two different forms of the potential (2.8), corresponding to the values $\lambda=1,2$. We report the results of the end-to-end distance for different values of $\lambda$ and for chains of increasing size $N$ at values of $\beta=0.5$ and $\beta=1.0$.

\begin{tabular}{|c|c|c|c|c|c|c|c|c|c|c|c|}
\hline$\lambda$ & $\beta$ & $\mathrm{N}=120$ & $\mathrm{~N}=240$ & $\mathrm{~N}=320$ & $\mathrm{~N}=520$ & $\mathrm{~N}=1024$ & $\mathrm{~N}=2048$ & $\mathrm{~N}=4096$ & $\mathrm{~N}=8192$ & $\mathrm{~N}=16384$ & $\mathrm{~N}=32768$ \\
\hline 1.0 & 0.5 & 60.6 & 128.2 & 174.5 & 293.2 & 601.7 & 1249.4 & 2583.4 & 5372.2 & 11071.2 & 22769.8 \\
\hline 1.0 & 1.0 & 78.0 & 164.4 & 223.6 & 374.2 & 767.59 & 1590.6 & 3283.9 & 6778.8 & 13942.4 & 28627.3 \\
\hline
\end{tabular}

\begin{tabular}{|c|c|c|c|c|c|c|}
\hline$\lambda$ & $\beta$ & $\mathrm{N}=120$ & $\mathrm{~N}=240$ & $\mathrm{~N}=320$ & $\mathrm{~N}=520$ & $\mathrm{~N}=1024$ \\
\hline 2.0 & 0.5 & 26.2 & 48.6 & 63.0 & 98.12 & 140.2 \\
\hline 2.0 & 1.0 & 33.2 & 62.5 & 81.4 & 126.2 & 248.7 \\
\hline
\end{tabular}

Tab.1: Results for the end-to-end distance $R$ for different values of the chain length $N$ and the interaction strength $\beta$ and $\lambda$. These results are obtained solving explicitly the the Euler equations (2.11)-(2.15).

Let us begin to discuss the case of a simple polyelectrolyte chain in $d=3$ with pure Coulombic interaction $(\lambda=1)$. We analyzed the end-to-end distance behavior for increasing values of $N$, i.e. $b(N / 2)$. This is reported in Fig.1. Dependence of the exponent $\gamma$ on the interaction strength suggests a weak violation of universality as manifest in Fig.2. 


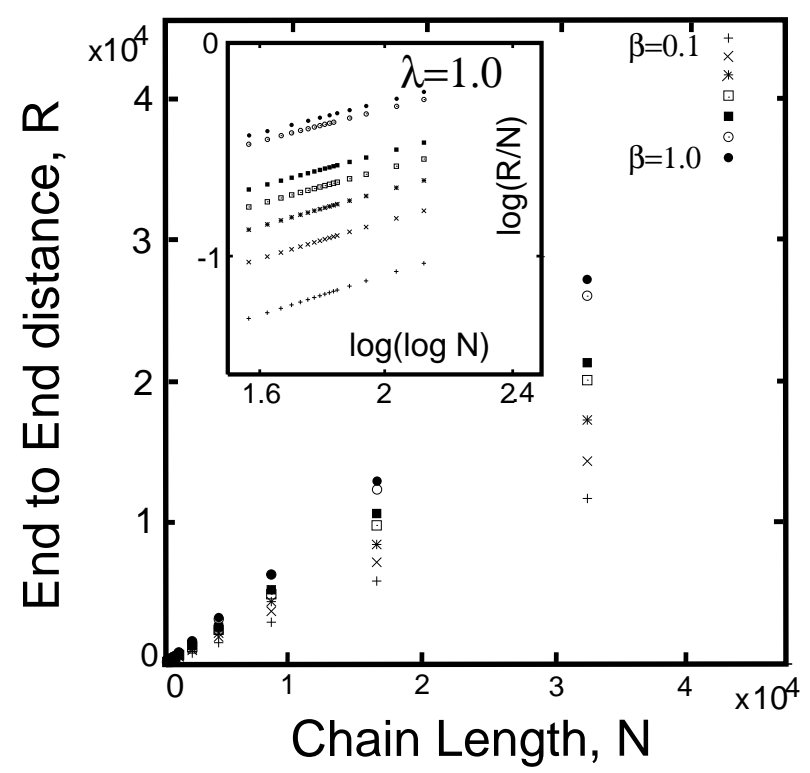

FIG. 1. End-to-End distance $R$ versus the chain length $N$, for increasing values of the interaction strength $\beta$. The inset shows the logarithmic plot, when a linear dependence of $R$ on the chain length is assumed, beside logarithmic corrections, for values of $\lambda=1$ in equation 2.8.

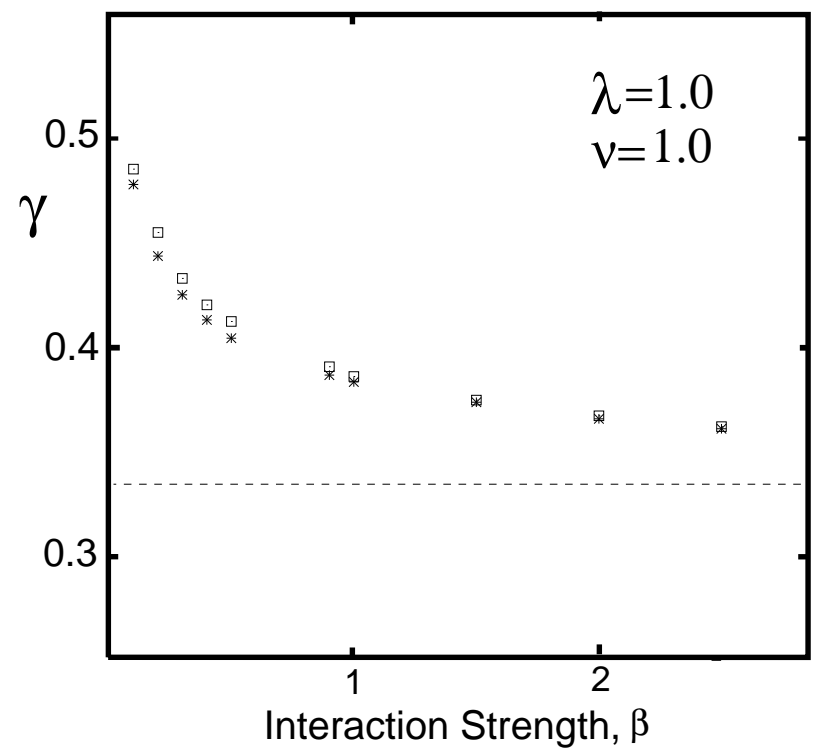

FIG. 2. The exponent $\gamma$ as measured (see Fig.1) for different values of the interaction strength $\beta$. The exponent $\gamma$ is seen to decrease for large values of the interaction strength, towards the expected value $\gamma=1 / 3$. The squares indicates the computed value of $\gamma$ with chains of length $N<1024$ while stars show the computed value of $\gamma$ for chains of length $N<2^{15}$.

The des Cloizeaux argument, once extended to the polyelectrolyte chain problem in $d=3$ implies an overstretching $(\nu=2 / \lambda=2)$, while the ansatz suggested above indicates $\nu=3 /(\lambda+2)=1$. In order to compute the non-universal exponent $\gamma$ we plot $R / N^{3 /(\lambda+2)}$ as shown in the inset of Fig.1. The nonuniversal behavior of $\gamma$ agrees with the inequality (2.42) derived in the asymptotic analysis of section II. In Fig.2, the exponent $\gamma$ decreases with increasing values of $\beta$, approaching values very close to $\gamma=1 / 3$, for large values of the interaction strength. A recent discussion about the logarithmic correction to scaling behavior for polyelectrolyte chains, has been given [19] and a direct derivation of the value $\gamma=1 / 3$ has been obtained. In the notation of reference [19] the small temperature limit $T \rightarrow 0$ corresponds to the large interaction strength limit $\beta \rightarrow \infty$ which is consistent with our findings. The result

$$
R \propto N(\log N)^{1 / 3}
$$


obtained in the large $\beta \rightarrow \infty$ limit, can be interpreted as a stretching of the harmonic bonds of the chain. The value of $\gamma=1 / 3$ was also obtained by a simplified chain under tension variational method [18] where the trial Hamiltonian is the Hamiltonian of a Gaussian chain subject to an external tension $\vec{F}$. This result, confirmed also by the Monte Carlo simulations presented in the next section, holds in the limit of large interaction strength but should not considered as a limiting case of little practical impact. Ordinary temperatures in the aqueous solutions correspond to surprisingly "high" values of the interaction strength $\beta$ 19.

A rather important problem discussed in the past [7] is the polyelectrolyte chain with an interaction (2.8) at $\lambda=2$. After the proper rescaling of the interaction strength $\beta$ (see equation 2.10), this problem can be thought as a chain with pure Coulombic interaction for $d=4$. Results for the end-to-end distance versus the chain length $N$ are shown in Fig.3.

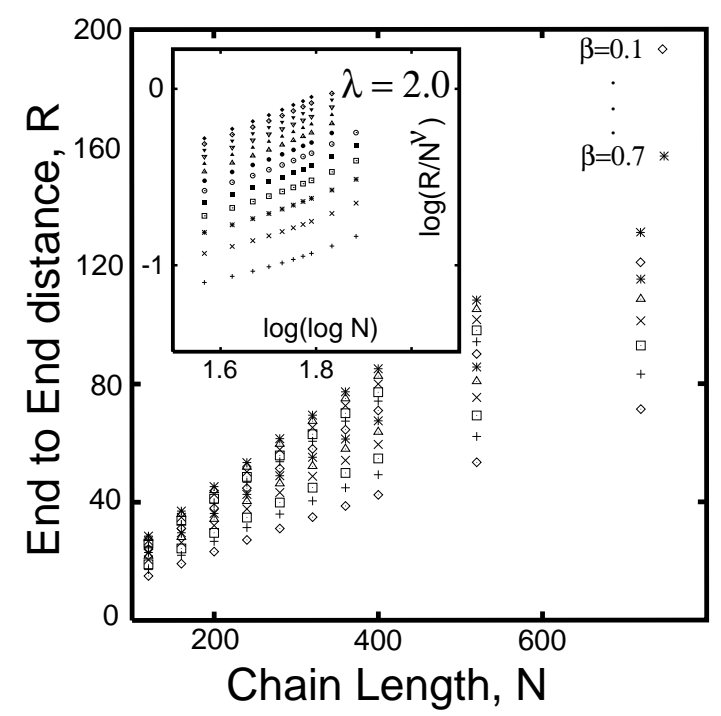

FIG. 3. End-to-End distance $R$ versus the chain length $N$ for increasing values of the interaction strength $\beta$ at $\lambda=2$. The inset shows the logarithmic plot, when a power law $N^{\nu_{F}}$ dependence of $R$ on the chain length is assumed, beside logarithmic corrections.

A similar discussion about the non-universal behavior of the exponent $\gamma$, follows the same lines as in the previous case (Fig.1-Fig.2). According to the ansatz we proposed in the asymptotic analysis of section II C we compute the exponent $\gamma$ as in Fig.4, where $\nu=\nu_{F}$ is the Flory exponent.

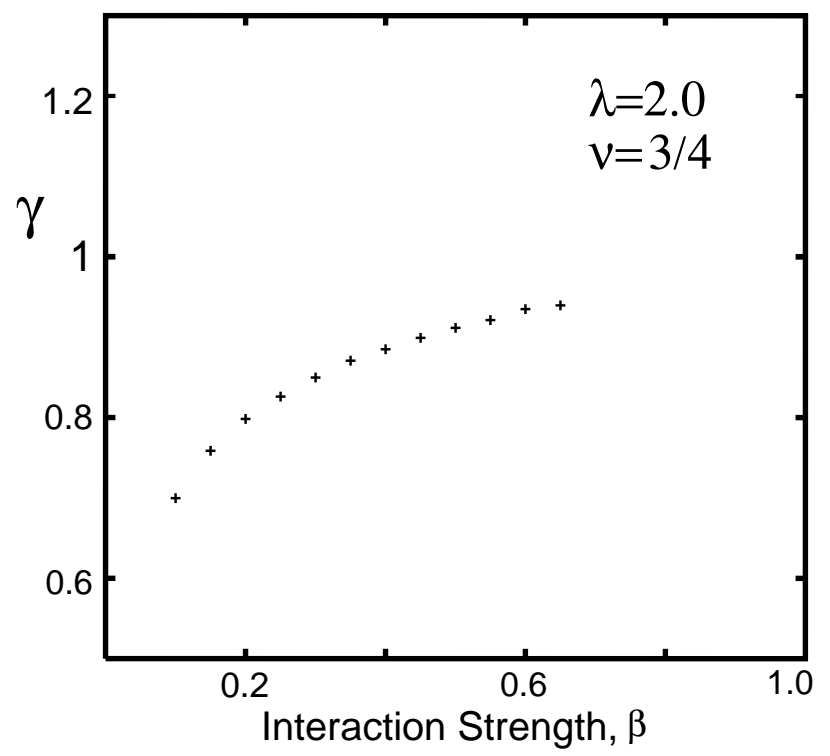

FIG. 4. The exponent $\gamma$ as measured from Fig.3 for different values of the interaction strength $\beta$. The exponent $\gamma$ is seen to increase for large values of the interaction strength towards the value $\gamma \approx 1.0$. 
In Fig.5 we compute the non-universal behavior of the exponent $\gamma$ assuming a linear dependence of the end-to-end distance of the chain length $N$, beside logarithmic terms. This is not the case, for the potential (2.8) with $\lambda=2$, but interesting comparison with previous results follows. The exponent $\gamma$, as in Fig. 5 , approaches values of $\gamma \approx-0.4$ at values of $\beta=1.0$ where Monte Carlo calculations were performed 22 and where the computed value of $\gamma \approx-0.35$ is in good agreement with our result. A two parameter fit would represent a very conclusive way to discriminate between these two possibilities, but the chain lengths that one can reach in simulations and in a direct minimization of the variational free energy are not long enough to make such analysis effective.

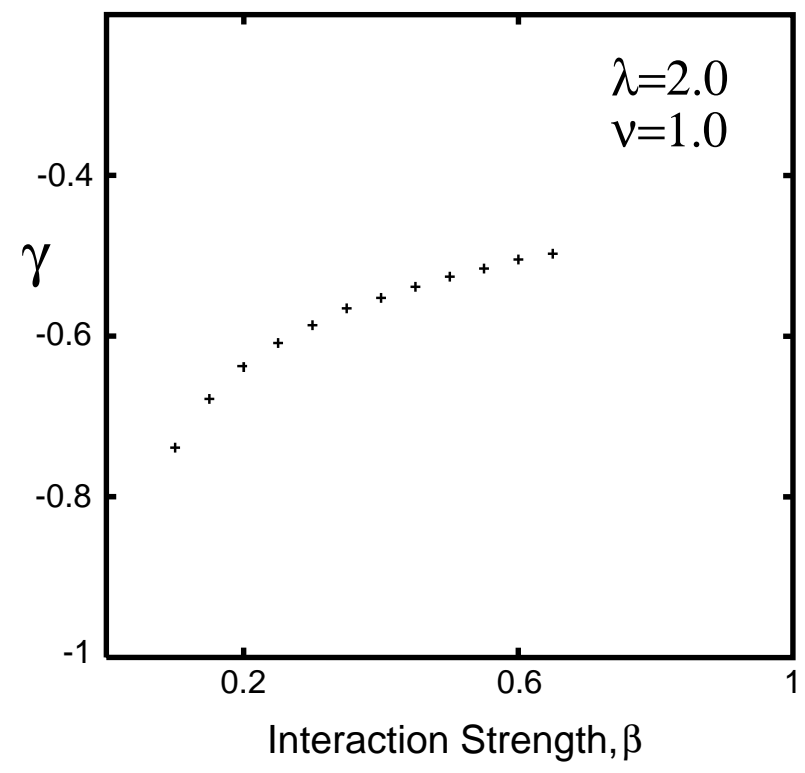

FIG. 5. The exponent $\gamma$ as measured from Fig.3 for different values of the interaction strength $\beta$. The exponent $\gamma$ is seen to increase for large values of the interaction strength towards the value $\gamma \approx 1.0$, once a linear dependence of $R$ on the chain length is assumed, beside logarithmic corrections, for values of $\lambda=2$ in equation (2.8). This results should be compared with the value of $\gamma \approx-0.35$ previously found via Monte Carlo simulations, for values of $\beta=1.0$.

\section{B. Electrostatic blob picture}

We will now present a numerical analysis of the electrostatic blob picture. We measured the crossover (shown by the mean square distance as a function of the polymer chain length $N$ ) from a Gaussian regime, observed at small distances, to the stretched regime for long enough distances. This represents an effective way to compute the electrostatic blob size and to check our method. One of the major achievement of the Gaussian variational approach discussed in section I is that it does not simply provides a quantitative description of the large $n$ behavior correlation function. A full numerical solution of the Euler equations (2.11)-(2.15) provides informations on the intermediate behavior of the correlation functions $b(n)=\left\langle\left(r_{i+n}-\vec{r}_{i}\right)^{2}\right\rangle$. That is why it is interesting to check whether short enough segments of the polymer chains behave as Gaussian chains, as expected from general scaling arguments [2], whereas the large $n$ behavior corresponds to equation (2.44). Once the crossover is observed, the size of an electrostatic blob $\xi$ can be extrapolated. The dependence of the electrostatic blob size $\xi$ from the interaction strength $\beta$, that we expect from phenomenological arguments, is indeed confirmed within our variational approach (see Fig.6). In particular we expect

$$
\begin{aligned}
& R \propto(N / g) \xi \simeq N a \beta^{1 / 3} \\
& \xi \propto a \beta^{-1 / 3}
\end{aligned}
$$

In Fig. 6 we show the electrostatic blob size as a function of the interaction strength $\beta$. The $\xi \propto \beta^{-1 / 3}$ behavior expected from standard scaling arguments is here also found. 


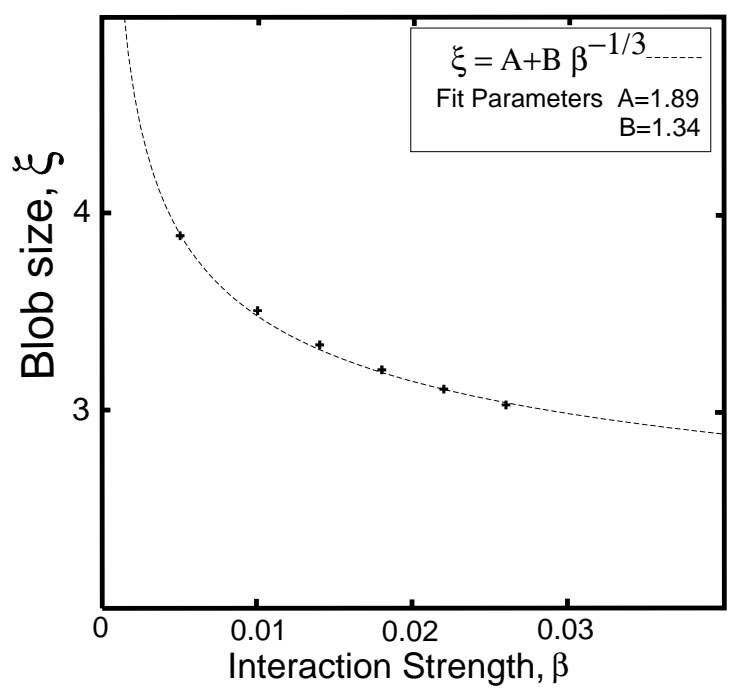

FIG. 6. Blob size, as measured from the direct solution of equations (2.15)-2.10, considering the crossover between the small distance behavior of the mean square distance $b(n)$ versus the large distance behavior. Different values of the electrostatic blob size for increasing values of the interaction strength $\beta$ are compared with the $\xi \approx \beta^{-1 / 3}$ behavior expected from phenomenological arguments.

\section{Results for the Self Avoiding Chain problem}

We consider the problem of a polymer chain with a short range interaction between the monomers, in a good solvent. Within the Gaussian Variational Principle, the expression for the free energy and the Euler equation are given by equations (2.16) and (2.18). In section II we have discussed the dimensional shift (2.17). Nevertheless this equivalence is purely formal and no deep insight is hidden behind it. The two problems considered are indeed pretty different and they reduce to the same type of variational equations because of the Gaussian approximation itself. It is important to remark that the Gaussian Variational Principle, specially reliable for the long ranged polyelectrolyte problem, has been originally introduced to study the self avoiding homopolymer chain problem [5.,6]. Even though it is nowadays well established that the Gaussian Variational Principle is not very well suited for the short ranged interacting chain problem, we wish to make clear that within this approximation, apart for spurious logarithmic corrections, the usual Flory-like power law behavior should be recovered.

In the original asymptotic analysis of des Cloizeaux the non integer value of $\delta$ is tacitly assumed, so that the result of $\nu=2 / d$ for the swelling exponent was obtained. Although we believe this result to be incorrect, it is often mentioned in the literature to discuss the asymptotic properties of polyelectrolyte chains [13]. According to our analysis and to the previous analysis of Allegra and coworkers [9], the problem of a self avoiding chain in good solvent turns out, within the Gaussian Variational Principle, to present the expected Flory-like behavior. This is not surprising since the original derivation of Flory also belong to the class of Gaussian Variational approximations, since a Gaussian probability distribution of the distance between the ends of the chain is assumed. A numerical justification of the des Cloizeaux result for the swelling exponent can also be found in the literature [8]. We believe that this numerical analysis is inconsistent since the constraint on the interaction parameter (assumed by des Cloizeaux and critically reviewed in section II) is enforced in the numerical algorithm. This makes the results obtained highly questionable. In Fig. 7 and Fig. 8 we present the results of the end-to-end distance for the self avoiding homopolymer chain problem studied via the numerical solution of the Euler equations discussed above. 


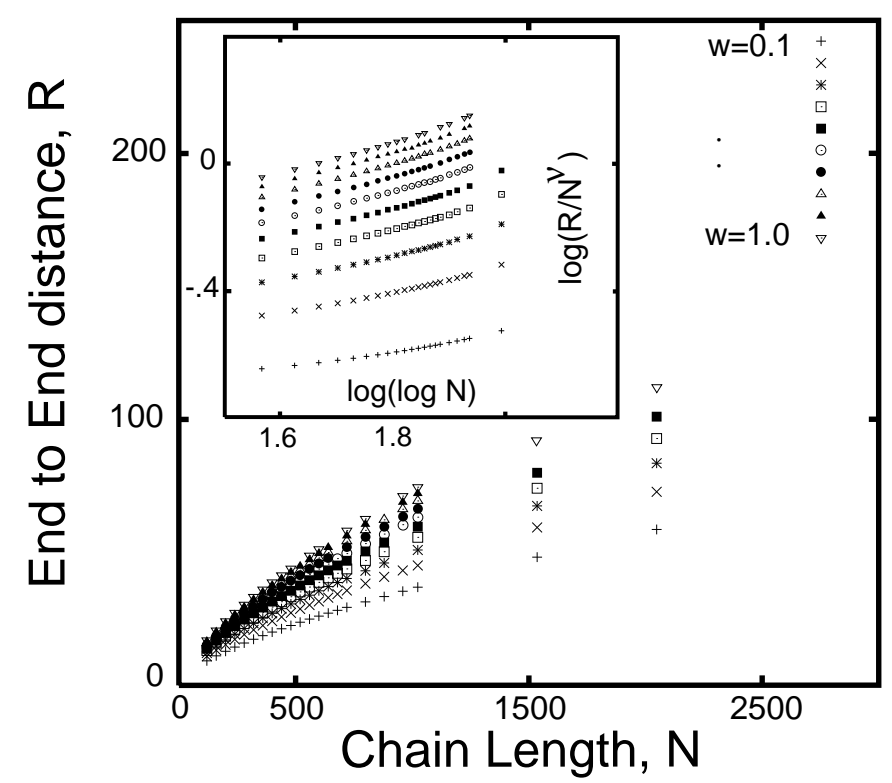

FIG. 7. End-to-End distance $R$ versus the chain length $N$. for increasing values of the interaction strength $w$, that represent in this case the quality of the solvent. The inset shows the logarithmic plot, when a $N^{\nu_{F}}$ dependence of $R$ on $N$ is assumed, beside logarithmic corrections.

Results for the end-to-end distance as a function of the polymer chain length $N$ are shown and a quantitative analysis of the non universal logarithmic part to be considered as a spurious effect due to the short range nature of the problem is presented.

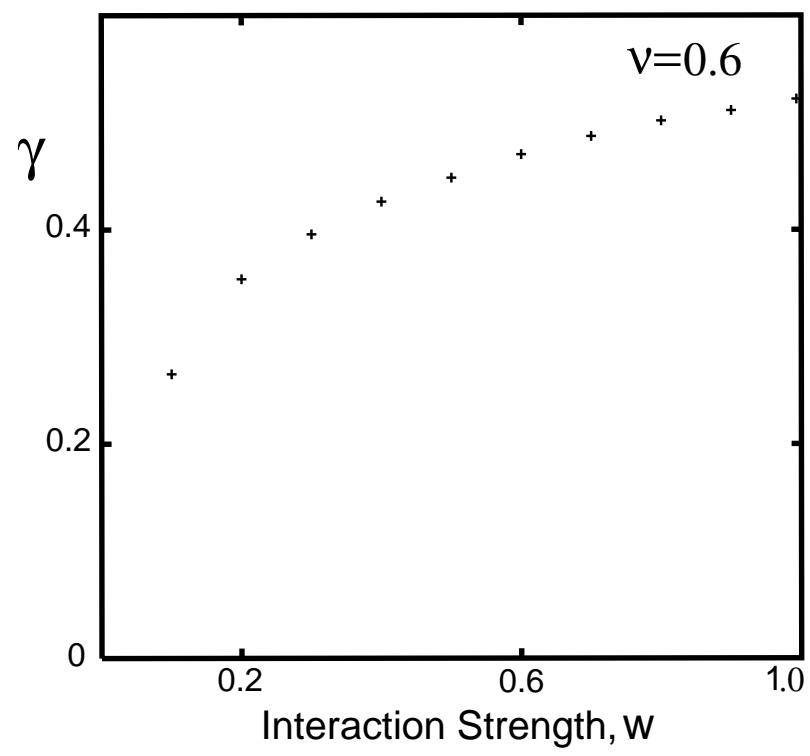

FIG. 8. The exponent $\gamma$ as measured from Fig.7 for different values of the interaction strength $w$. The exponent $\gamma$ is seen to increase for large values of the interaction strength.

According to the asymptotic analysis given above we expect the exponent $\gamma$ to be non universal and to depend indeed on the quality of the solvent.

\section{NUMERICAL SIMULATIONS}

In order to test the conclusions obtained in the previous section we decided to perform a numerical simulation for a polyelectrolyte chain in an ideal salt free solution, when pure Coulombic interactions are considered between the monomers. A bit of caution is required since we will compare the results of our 
simulations with the results obtained via the Gaussian variational principle, where a cyclic geometry for the polymer chain is chosen. It was easy however to device a numerical simulation of the Monte Carlo type where close chain configurations can be taken under investigation. As remarked long time ago, [6] the cyclic geometry chosen within the variational calculation becomes unessential as soon as long enough chains are considered and this turns out to be confirmed by our numerical simulations. We then consider a Monte Carlo simulation, performed in the canonical ensemble via the standard Metropolis algorithm. We consider a pivot move for what concerns the elementary Monte Carlo move, i.e. we assume rigid bonds between the monomers and an elementary pivot move consists in a random rigid rotation of a randomly chosen part of the chain. The pivot algorithm, first described in reference [20] and lately applied in the context of polyelectrolyte chains with and without salt [19] is a very effective method to confirm our general conclusions. Several million Monte Carlo moves (the number which was properly increased with the chain length) are required in order to reduce below $1 \%$ percent the statistical fluctuations for the end-to-end distance. We also combine our Monte Carlo simulation with a Molecular dynamics subroutine that is randomly called, during the Monte Carlo simulation, with a certain fractional occurrence ratio. On the one side, the pivot algorithm is well suited to simulate the polymer chain at long enough scales. The pivot moves are in a sense collective moves and a good description of the chain is certainly obtained at long scales. On the other side the Molecular Dynamic algorithm is well suited to simulate the chain at small distances. We initially perform independent simulations with the Monte Carlo and the Molecular dynamics algorithm in order to tune the simulation parameters involved for a chain of a given length $N$. Once the two methods were independently tested, we proceeded to combine them as discussed above.

We now present our results for the end-to-end distance for $d=3$ polyelectrolyte problem. In Fig. 9 the end-to-end distance is shown as a function of the polymer length $N$ for the specific value of $\beta=1.0$. The inset shows the end-to-end distance divided by the pure power law Flory-like dependence $N^{\nu}$ as a function of $\log N$. We repeated the same analysis of Fig.9 changing the value of the interaction strength $\beta$. The non universal behavior obtained in the asymptotic analysis of section II and in the numerical analysis of the Euler equations (2.11) - (2.15), is found within our simulation results (see Fig.10).

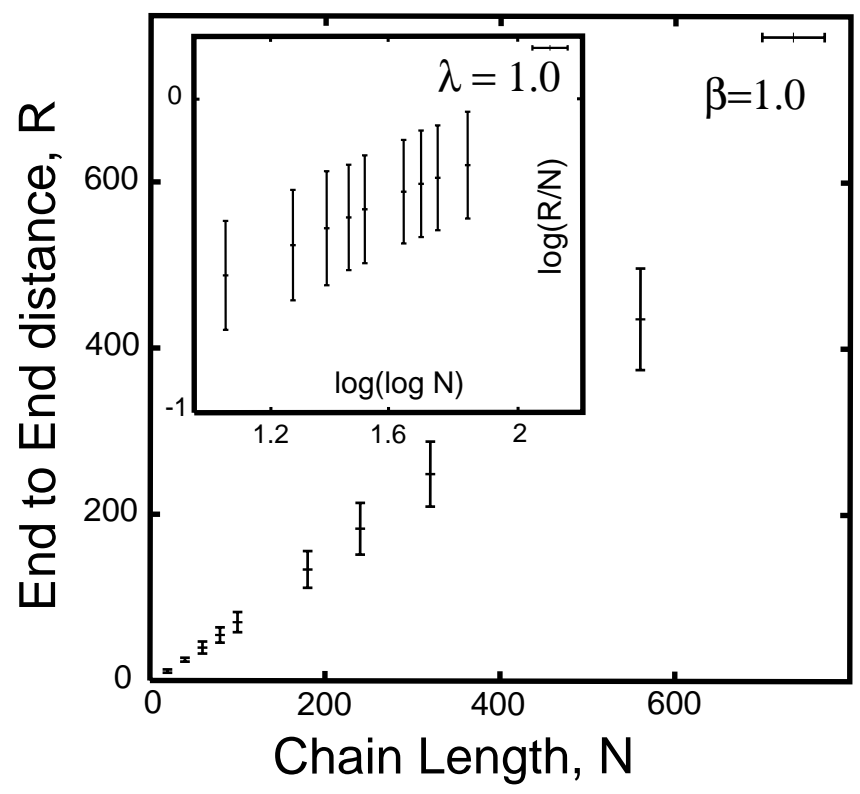

FIG. 9. End-to-End distance $R$ versus the chain length $N$ for the polyelectrolyte chain at increasing values of the interaction strength $\beta$ and $d=3$, obtained via the pivot Monte Carlo algorithm combined with Molecular Dynamics for the value of the occurrence factor $r=0.1$. Different simulations, corresponding to different values of the occurrence factor $r$ have been shown to be numerically consistent within each other. The inset shows the logarithmic plot, when a linear dependence of $R$ on $N$ is assumed, beside logarithmic correction

The non universal exponent $\gamma$ is seen to decrease, as we obtained with the variational method of section II-III. Nevertheless one should treat the results of simulation with a bit caution. 


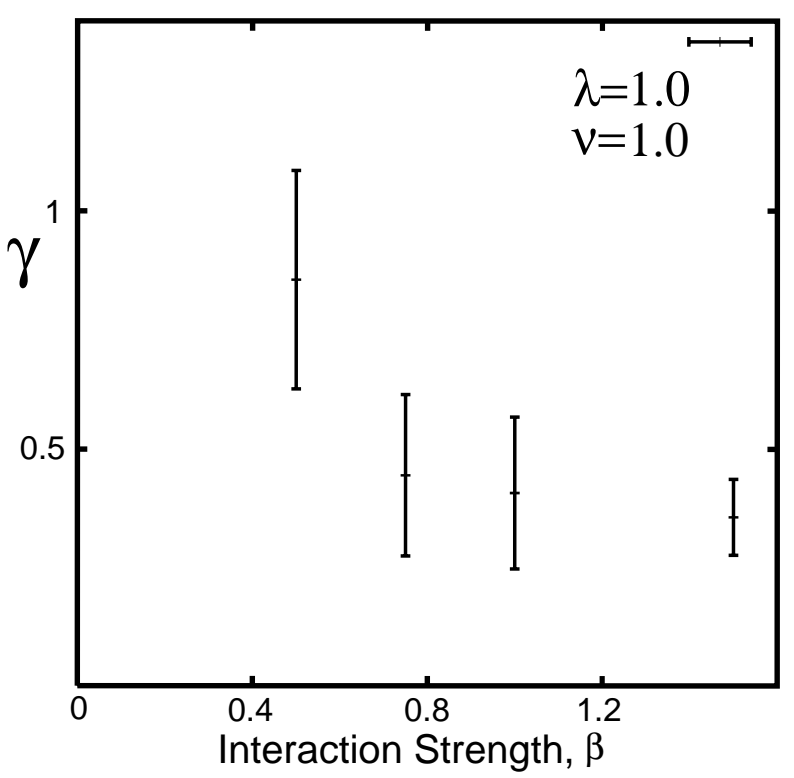

FIG. 10. The non universal exponent $\gamma$, for different values of the interaction strength $\beta$ as measured from Fig.9, for the specific value of $\beta=1.0$ and for the values $\beta=0.5,0.75,1.5$.

In the numerical simulations monomers are considered to be rigidly connected while in the variational principle approach monomers are connected by springs. In this case one can not expect that the exponent $\gamma$ as a function of interaction strength has the same large $\beta$ behavior.

\section{CONCLUSIONS}

In the present paper we have reinvestigated the Gaussian variational principle and its applicability to polymer chain with long and short range interactions. The asymptotic analysis originally presented by des Cloizeaux in the context of self avoiding walks has been critically reconsidered. In particularly we have shown that the des Cloizeaux's analysis overconstains the model's parameters. It has been found that within the Flory type of balancing conditions (when the elastic term is balanced by the interaction but the entropic term is not relevant) the asymptotic law (2.33) satisfies the Euler equation (2.15) . In this asymptotic law the Flory exponent $\nu=3 /(d+2)$ and logarithmic corrections to scaling is characterized by a non - universal exponent $\gamma$. Within the Gaussian variational principle the universal exponents for the case of the Coulombic and short range interactions are related by a dimensional shift (2.17).

Gaussian variational principle suits much better for the long range problems which was already discussed in reference [7]. The arguments we have presented prove that the scaling form (2.33) is valid for a general long - range interaction potential (2.8). The exponents $\nu$ and $\gamma$ satisfy the equations (2.41) - (2.42). According to the condition (2.42), the exponent $\gamma$ is non - universal and depends from the interaction parameter as well as from the chain model (compare e.g. Fig.2 and Fig. 10). In this sense we may consider the combination $l_{\text {eff }}=a(\log N)^{\gamma}$ as an effective Kuhn segment for chains with unscreened type of interactions.

On the contrary, for the short range interactions the Gaussian variational principle simply overestimates the probability of the segment - to - segment contacts [7] which leads to more extended configurations compare to the Monte Carlo results [20]. We conclude observing that the models which are characterized by the interplay between long - ranged attraction and repulsion are also useful systems to be treated by the Gaussian variational principle, as presented here. Appropriate systems are polyampholyte [26]. For the randomly charged polyampholytes Kantor and Kardar 27 have found strongly nonuniversal behavior. For example at $d<4$ the spatial configurations depend on the polyampholyte excess charge $Q$ so that if $Q>Q^{*} \approx e \sqrt{N}$ the compact form changes to the stretched one. We believe that the Gaussian variational principle is a good starting point for the investigation of such systems.

\section{APPENDIX A}

The asymptotic analysis of equations (2.18) suggests to consider the following series 


$$
S(\delta, z)=\sum_{n=1}^{\infty} \frac{z^{n}}{n^{\delta}}
$$

In the asymptotic limit $k \rightarrow 0$ the interaction term of equation (2.18) is simply related to $S(\delta, z)$. Even the simple power law ansatz $b(n) \propto n^{\nu}$ requires some care. The expression (5.1) is evaluated exactly but two rather different expressions are obtained if the exponent $\delta=\nu(d+2)$ is assumed to be integer or non-integer. The case of the exponent $\delta$ being non-integer reduces the interaction term to the expression given in equation (2.25) and leads to the des Cloizeaux result. Assuming an integer value for the exponent $\delta$ produces instead a different expression for the interaction term and the proper balancing condition with the elastic energy naturally follows.

Let us consider the series $S(\delta, z)$ in this two distinct cases. In equation (5.1) $z$ and $\delta$ can be complex quantities, as soon as $|z| \leq 1$ and $\operatorname{Re} \delta>1$ at $|z|=1$ whereas $\operatorname{Re} \delta>0$ if $|z|<1$. The calculation of the series (5.1) ( see sec. 1.11 in ref. [16] ) gives

$$
S(\delta, z)=\Gamma(1-\delta)\left[\log \left(\frac{1}{z}\right)\right]^{\delta-1}+\sum_{r=0}^{\infty} \zeta(\delta-r) \frac{(\log z)^{r}}{r !},
$$

where $|\log z|<2 \pi, \Gamma(x)$ is the gamma function, $\delta \neq 1,2, .$. and $\zeta(x)$ is the $\zeta$-zeta Riemann's function. We are interested in the case of $z=\exp (i k)$ at $k \rightarrow 0$. Considering the real part of both sides of equation (2.23) in the asymptotic limit $k \rightarrow 0$, we obtain equation (2.25).

We now consider the case of integer values of $\delta$. The $\Gamma(x)$ has poles at all negative integer arguments whereas the pole of $\zeta(x)$ is placed at $x=1$. We write $\delta=m+\epsilon$ where $m$ is a positive integer and $\epsilon \rightarrow 0$. Then in the vicinity of poles the gamma and $\zeta$ - functions can be rewritten as

$$
\begin{aligned}
\Gamma(1-m-\epsilon) & =\frac{(-1)^{m}}{(m-1) !}\left\{\frac{1}{\epsilon}-\psi(m)+\mathcal{O}(\epsilon)\right\} \\
\zeta(1+\epsilon) & =\left\{\frac{1}{\epsilon}-\psi(1)+\mathcal{O}(\epsilon)\right\} .
\end{aligned}
$$

We should also take into account that

$$
\left[\log \left(\frac{1}{z}\right)\right]^{\epsilon}=1+\epsilon \log \log \left(\frac{1}{z}\right)+\mathcal{O}\left(\epsilon^{2}\right) .
$$

After that the equation (5.2), due to the cancellation of poles in gamma and $\zeta$ - functions at small values of $\epsilon$, becomes [16]

$$
\begin{aligned}
S(m, z) & =\frac{(\log z)^{m-1}}{(m-1) !}(\psi(m)-\psi(1)-\log \log (1 / z)) \\
& +\sum_{r=0}^{\infty} \zeta(m-r) \frac{(\log z)^{r}}{r !}
\end{aligned}
$$

where the prime indicates that the term $r=m-1$ is to be omitted. In equations (5.5) (5.3) $\psi(x)$ is the digamma function, defined as the logarithmic derivative of the gamma function $\psi(\delta)=\frac{d \log \Gamma(\delta)}{d \delta}$. In the limit $k \rightarrow 0$, for $z=\exp (i k)$ and $\delta=3$, eq. (5.5) have been considered in eq.(2.31).

We want to show now that the integral representation of the sum is correct in the asymptotic limit and that finite $n<l$ terms do not contribute in this limit. Let us consider explicitly the presence of a finite cutoff in the series (5.1).

$$
S(\delta, z)=\sum_{n=l}^{\infty} \frac{z^{n}}{n^{\delta}},
$$

which reduces to equation (5.1) at $l=1$. For integer values of $\delta$ it is easy to derive exactly the following expression [16]:

$$
\begin{aligned}
S(m, z, l) & =\frac{(\log z)^{m-1}}{(m-1) !}(\psi(m)-\psi(l)-\log \log (1 / z)) \\
& +\sum_{r=0}^{\infty} \zeta(m-r) \frac{(\log z)^{r}}{r !} .
\end{aligned}
$$


Consider the series (5.6) with a finite cutoff $l$ in the asymptotic limit $k \rightarrow 0$. We want to evaluate the contributions to the series from finite $n<l$ terms, when $m=3$. We obtain

$$
\sum_{n=l}^{\infty} \frac{1-\cos (n k)}{n^{3}} \simeq \frac{k^{2}}{2}(\psi(3)-\psi(l)-\log k)+O\left(k^{4}\right),
$$

so that the contribution from finite $n$ takes the following form

$$
\sum_{n=1}^{l} \frac{1-\cos (n k)}{n^{3}} \simeq \frac{k^{2}}{2}(\psi(l)-\psi(1))
$$

Summing up equations (5.8) and (5.9) we obtain again the result (2.31). The expression (5.8) shows that for small $k$ values only large $n$ terms contribute to the sum (5.6). Indeed the contribution coming from $n>l$ produces a term $k^{2} \log (k)$. This, whenever $k<<\exp (-\psi(l))$, will dominate the simple $k^{2}$ behavior. Moreover, for large values of $l$ (see ref. [16]) we have $\psi(l) \propto \log (l)$ and the condition above takes the form $k<<1 / l$. It follows that for $k<<1 / l$ the term $k^{2} \log (k)$ dominates and the sum does not depends on the low limit value. In this case, the series can be well approximated by an integral, i.e.

$$
S(k)=\sum_{n=l}^{\infty} \frac{1-\cos (n k)}{n^{3}} \simeq \int_{l}^{\infty} d n \frac{1-\cos (n k)}{n^{3}}=k^{2} \int_{k l}^{2 \pi} d x \frac{1-\cos (x)}{x^{3}}=k^{2} I(k)
$$

In order to compute $I(k)$, consider the derivative of the previous expression, $I^{\prime}(k l)=-1 / 2 k l$ so that $I(k l) \simeq-\frac{1}{2} \log (k l)+c$, where $c$ is a constant, and $S(k)=\frac{k^{2}}{2}(c-\log (k l))+O\left(k^{4}\right)$. Comparison with equation (5.8) shows that for $k<<1 / l$ only large $n$ contributions are relevant.

\section{ACKNOWLEDGMENTS}

We thank Christian Holm, Burkhard Dünweg and Ralf Everaers for continuous and fruitful discussions. In particular we thank Dr. Holm for several comments on the simulation part. One of us (G.M.) wish to express many thanks to Giorgio Parisi and Roland Netz.

[1] P. G. de Gennes Scaling Concepts in Polymer Physics, Cornell University Press: Ithaca, NY (1979).

[2] P.G. de Gennes, P. Pincus, R.M. Velasco, F. Brochard, J. Physique 37, 1461 (1976).

[3] J. L. Joanny and J. F. Barrat, Advances in Chemical Physics, (1995).

[4] G.S. Manning, J. Chem. Phys. 51954 (1969).

[5] J. des Cloizeaux, J. Physique 31, 715 (1970).

[6] J. des Cloizeaux G. Jannik, Polymer in Solutions, Clarendon Press: Oxford, U.K. (1990).

[7] J. P. Bouchaud, M. Mézard, G. Parisi and J. S. Yedida, J. Phys. A24, L1025 (1991).

[8] D. Bratko and K.A. Dawson J. Chem. Phys. 99, 5352 (1993).

[9] G. Allegra and F. Ganazzoli, J. Chem. Phys 76 6354,(1982).

[10] E. Shakhnovich A. M. Gutin, J. Phys. A 22 1647, (1989).

[11] T. Garel H. Orland Europhys. Lett., 6 (4), 307 (1989).

[12] M. Mézard, G. Parisi J. Physique 1, 809 (1991).

[13] R. Netz and H. Orland Eur. Phys. J.B 8 81, (1999).

[14] G. Migliorini, to be published.

[15] C. Petersen, O. Sommelius and B. Söderberg, Phys. Rev. Lett. 76, 1079 (1996); B. Jönsson, C. Petersen and B. Söderberg, Phys.Rev.Lett. 71, (376) (1993).

[16] A. Erdélyi, Higher Transcendental Functions, vol.I,McGraw-Hill, New York (1953).

[17] W. Press W. Vetterling, Numerical Recepies, Cambridge University Press, New York (1985).

[18] J. L. Barrat D. Boyer, J. Physique 3 343, (1993).

[19] C. Petersen, B. Jönsonn and B. Söderberg, J. Phys. Chem., 99 (4), 1251 (1995).

[20] A. D. Sokal N. Madras, J. Stat. Phys. 50, 109 (1988); A. BaumgärtnerJ. Phys. (Paris) 45 L515, (1984).

[21] G. Allegra and F. Ganazzoli, J. Chem. Phys 87 1817,(1987). 
[22] E. Marinari G. Parisi Europhys. Lett., 15 (7), 721 (1991).

[23] P. Pfeuty, R. Velasco P.G. de Gennes, J. Physique Lett. 38, L5 (1977).

[24] A. Falicov A.N. Berker, Phys. Rev.. B 51, 12458 (1995).

[25] G. Migliorini A. N. Berker Phys. Rev. B 57, 426 (1998).

[26] P.G. Higgs and J.- F. Joanny J.Chem.Phys.94 1543 (1991).

[27] Y. Kantor and M. Kardar Phys.Rev. E 51, 1299 (1995);Phys.Rev. E 52, 835 (1995) 